\title{
The Subsidiarity Bias in Regulation
}

\author{
Jean-Jacques Laffont* \\ and \\ Jérôme Pouyet ${ }^{\dagger}$
}

June, 2000

*IDEI and GREMAQ (UMR 5603 CNRS), Université des Sciences Sociales, Place Anatole-France, 31042 Toulouse Cedex, France.

${ }^{\dagger}$ CERAS-ENPC (URA 2036 CNRS), 28 rue des Saint Pères, 75007 Paris, France. E-mail: pouyet@enpc.fr 


\begin{abstract}
We study the choice of the regulatory structure when a regulated firm engages in different activities for different countries. Under decentralization each activity is regulated independently and the contracts offered to the firm suffer from two opposite distortions with respect to centralization: the competition between regulatory authorities forces them to offer too high-powered incentive contracts; however, because the ownership structure of the firm is dispersed across the countries, each regulator does not fully internalize the effect of his regulation on the firm's rent and contracts tend to be too low-powered. When the activities of the firm are sufficiently substitutable we show that decentralization always leads to an inefficient drift of the regulatory contracts towards fixed-price contracts. Nonetheless, when regulators have private agendas and possess the discretion to distort their policy to gain the support of some interest groups, then decentralization of the regulatory powers may be preferred to centralization as competition between regulatory authorities eradicates their discretionary power.
\end{abstract}

JEL Classification: D72, H41, H70, L20.

Keywords: incentives, decentralization, regulation. 


\section{Introduction}

What is the proper level of decentralization for public policy and in particular regulation? This question is very lively debated in federal states such as the USA or Brazil, as well as in the European Union. It is a special case of a more general debate about the desirability of multiple governments, with spatial specialization when we deal with decentralization, with domain specialization when we are concerned with a regulator per industry, or with functional specialization when we discuss the separation of regulation and competition policy. In Europe, the concept of subsidiarity has been put forward to express the idea that decentralization is desirable unless it entails too high coordination costs.

The optimality of the decentralization of public decision-making is an empty question in a world of complete contracts with benevolent decision-makers. Indeed, in such a setting, a centralized organization can always replicate the outcome of a decentralized one. We must introduce a degree of incompleteness (in the informational structures, in the sets of instruments or in the objectives) to create a trade-off between centralization and decentralization. Some recent papers have discussed this trade-off with a clear view of its foundation in terms of contractual limitations. ${ }^{1}$

In Caillaud, Jullien and Picard (1996) the focus is on the decentralization of industrial policies from the European level to the national level. If some variables are more likely to be observed at the national level they show that it is always optimal to decentralize part of the activities even in the presence of externalities between countries. Seabright (1996) introduces the notion of accountability to justify the possible superiority of decentralization. In his model, decentralization increases the accountability of the politicians in charge of decision-making and this effect can balance the non internalized externalities. Klibanoff and Poitevin (1997) rely on the lack of commitment power of the central government to favor decentralization which induces a direct bargaining between regions. Also, Olson and Torsvick (1993) and Martimort (1999) show that several regulators who leave more rents to the regulated agent carrying substitute activities is a commitment device. Laffont and Martimort (1998) show that the threat of collusion may lead the central government to delegate its authority when communication constraints alone would not yield this result. Laffont and Zantman (1999) base the trade-off on the better informational

\footnotetext{
${ }^{1}$ See also Sah and Stiglitz (1986), Hart and Moore (1999), Gilbert and Picard (1996) for organizational theories based on bounded rationality or implicit communication costs.
} 
structures of local politicians which are the joint products of local politics. Dewatripont and Tirole (1999) and Laffont and Martimort (1998) show in different contexts how a duality of regulators or supervisors is useful to provide incentives for regulators in charge of tasks which create negative externalities the ones on the others.

In this paper we develop a simple regulatory model to debate some pros and cons of decentralization or subsidiarity for the regulation of natural monopolies. Local favoritism, multiprincipal externalities and political economy under incomplete information are the main ingredients of the trade-offs we study. More precisely, we use the regulatory setting of Laffont and Tirole (1993) in which a firm is in charge of two procurement activities. ${ }^{2}$ Each regulator wants the firm to realize a country-specific project, and each project requires a specific effort from the firm which has private information about its cost characteristics. This informational advantage yields an (information) rent to the firm.

Under centralization, a unique regulator coordinates both decisions, whereas under decentralization each activity is regulated independently. With benevolent regulators suffering from asymmetric information with respect to the firm, decentralization suffers from two distortions. The first one is related to the multiprincipal design of the model. Because the actions taken by the firms are substitutes, each regulator is led to increase the effort he requires from the firm in equilibrium: this is the competition effect. The second effect is due to our specification of the ownership structure of the firm. We assume that in each country some of the citizens hold some shares in the firm. Hence, the rent of the firm goes back to the shareholders of each country. Under centralization, the regulator takes into account the effect of his regulation on the whole rent of the firm that belongs to the consumers of both countries. However, under decentralization, each regulator cares only about the consumers and shareholders of his country. As a result, decentralization leads the regulators to induce a too low effort level: this is the shared-rent externality.

When efforts are sufficiently substitutable we show that the competition effect is dominant and in the limit this can lead the regulators to offer fixed-price contracts in equilibrium: decentralization makes rent extraction impossible and the firm earns a large rent from the non coordination of the regulations.

Next, we consider that regulators may be captured. As in Laffont (1996) we consider a random majority model and assume that the regulators act in favor of the majority in

\footnotetext{
${ }^{2}$ Similar results could be obtained with a regulation model with variable quantities as Laffont-Tirole (1986) as well as with oligopolistic industries such as Auriol-Laffont (1992).
} 
power. In this case, we show that decentralization might be preferred as it reduces the discretionary power of the decision-makers.

The structure of the paper is as follows. The next section introduces the model with benevolent regulators. In Section 3, we show that decentralization is equivalent to centralization when regulators are under complete information vis-à-vis the firm. In Sections 4 and 5 we compare centralization and decentralization under asymmetric information. Section 6 does the same comparison when the objectives of the regulators are biased in favor of some citizens. Section 7 concludes. All the proofs are gathered in appendices.

\section{The model}

We take a partial equilibrium approach and consider two countries (or regions) $i=1,2$ in which a firm is realizing a project with (gross) value $S_{i}$ for the consumers of country $i^{3}$.

The firm can provide an effort $e_{i}$ in order to reduce the cost associated with project $i$. The cost function of the firm for project $i$ is $C_{i}=\beta-e_{i}$ where $\beta$ is the intrinsic efficiency parameter of the firm. We assume that the efficiency of the firm is the same for both projects. Parameter $\beta$ can take values in $[\underline{\beta}, \bar{\beta}]$ according to a common knowledge probability distribution with density $f($.$) and \operatorname{cdf} F($.$) satisfying the monotone hazard rate$ condition $\left(\frac{d}{d \beta} \frac{F(\beta)}{f(\beta)} \geq 0\right)$. In order to obtain explicit solutions we will sometimes illustrate our solutions in the case of a uniform distribution on $[0,1]$.

The cost reducing efforts create a disutility to the firm equal to

$$
\psi\left(e_{1}, e_{2}\right)=\frac{1}{2}\left(e_{1}^{2}+e_{2}^{2}\right)+\gamma e_{1} e_{2} .
$$

We assume that $\frac{\partial^{2} \psi}{\partial e_{1} \partial e_{2}}=\gamma>0$, or equivalently that the two efforts are substitutes from the point of view of the firm. Note that the firm cannot manipulate costs ${ }^{4}$. Accounting separation can be perfectly implemented but the firm can decide to allocate unobservable effort in a way that maximizes its rent. Parameter $\gamma$ belongs to $[0,1]$ and a high value of this substitutability index means that effort can be easily substituted from one project to the other (and conversely). Note that the disutility function is increasing and convex in both efforts. We also assume that regulator $i$, denoted by $P_{i}$, fully reimburses the

\footnotetext{
${ }^{3}$ Throughout the paper, we will assume that $S_{i}$ is sufficiently large so that each regulator does not want to shut down the realization of the project for some types of firm.

${ }^{4}$ See Laffont and Tirole (1993), chapter 12, for a model of regulation with cost padding.
} 
(observable) cost $C_{i}$ of activity $i^{5}$ and does not observe the realized cost on the other activity. The gain of the firm is then given by

$$
U=t_{1}+t_{2}-\psi\left(e_{1}, e_{2}\right)
$$

where $t_{i}$ is the net transfer given by $P_{i}$.

In country $i, P_{i}$ contracts with the firm for the realization of the (country specific) project. When the regulatory structure is splitted like that, we assume that the contracts are secret $\left(P_{i}\right.$ does not observe the contract proposed by regulator $P_{j}$ to the firm), and that regulators offer simultaneously contracts to the firm.

Each regulator must finance the realization of his project. In our partial equilibrium approach, the shadow cost of public funds $\lambda>0$ captures the distortionary effects of taxation $^{6}$. Regulator $P_{i}$ maximizes the welfare in country $i$, equal to the net surplus of the consumers/taxpayers plus a (so far) arbitrary sharing of the firm's rent, given by

$$
S W_{i}=S_{i}-(1+\lambda)\left(t_{i}+C_{i}\right)+\delta_{i} U \quad i=1,2
$$

with $\delta_{1}+\delta_{2}=1$. These shares reflect the distribution of ownership between the consumers of the two countries. We assume that $1+\lambda-\delta_{i}>0, i=1,2$, for rent extraction to be desirable in both countries. Otherwise we would have to take into account individual rationality constraints of consumers.

As is usual in the multiprincipal literature, we assume that if the firm decides to realize a project, it must also realize the other project. If it refuses to participate at all then the firm receives a reservation utility normalized to $0^{7}$.

\section{$3 \quad$ Full information benchmarks}

In this section, we assume that the firm's efficiency is publicly known; this implies that the effort provided by the firm is also observable. We start with the case of a common regulator (centralization) and then proceed with the situation where the two regulators behave in a non cooperative way (decentralization).

\footnotetext{
${ }^{5}$ This is just an accounting convention.

${ }^{6} \mathrm{We}$ assume it is the same for both countries.

${ }^{7}$ This is the intrinsic common agency setting as coined by Bernheim and Whinston (1986).
} 


\subsection{Centralized regulation}

In this situation, a single regulator called $P_{c}$ wants to maximize the sum of the welfares in the two countries. Since he knows the efficiency parameter of the firm, he has only to ensure that the firm is willing to participate. In other words, this regulator solves the following program

$$
\left\{\begin{array}{l}
\max _{\left\{t, e_{1}, e_{2}\right\}} \sum_{i=1}^{2} S W_{i} \\
\text { subject to } U \geq 0 \quad \forall \beta \in[\underline{\beta}, \bar{\beta}] .
\end{array}\right.
$$

Immediate algebra yields the solution to this program:

Proposition 1 Under centralization and complete information the optimal levels of effort are symmetric ${ }^{8}$ and are given by

$$
e_{1}(\beta)=e_{2}(\beta)=e_{*}(\beta)=\frac{1}{1+\gamma}
$$

Moreover, the firm gets no rent.

The intuition is clear: the marginal disutility of each effort must be equal to its marginal cost saving effect. Because the public funds are costly it is optimal to leave no rent to the firm: the (unique) transfer is designed in such a way that the rent of the firm is equal to its reservation utility.

\subsection{Decentralized regulation}

When each regulator $P_{i}$ knows the private information of the firm and when regulators behave in a non cooperative way, we are back to the previous situation. Indeed, each regulator can make the firm residual claimant of their relation, whatever the contract proposed to the firm by the other regulator. We conclude this subsection with the following proposition.

Proposition 2 Under complete information, decentralization is equivalent to centralization $^{9}$.

\footnotetext{
${ }^{8}$ Under centralization, when $\gamma=1$ only the sum of the efforts is determined in equilibrium. This holds under complete and incomplete information.
} 
The coordination between regulators on how to share the payments to the firm is not described by the model: only the sum of the transfers is determined ${ }^{10}$.

\section{Centralized regulation under asymmetric informa- tion}

Asymmetric information has been recognized as being a major obstacle to first-best efficient regulation. Following the new regulatory economics, we model the regulatory process as a principal-agent problem in which the firm has a superior knowledge on its efficiency.

When the two regulators cooperate perfectly the problem is equivalent to a usual adverse selection problem with a two-dimensional action ${ }^{11}$. According to the Revelation Principle $^{12}$, we can restrict ourselves to direct and truthful contracts: the outcome of any regulation stipulating a transfer depending on the realized costs can be replicated by a regulatory contract in which the firm reveals truthfully its private information. These additional incentive compatibility constraints will undermine the efficiency of the regulation and force the regulators to move away from the first-best (full information) contract.

Let us now determine the requirements of incentive compatibility. We denote by

$$
U(\beta ; \tilde{\beta})=t(\tilde{\beta})-\frac{1}{2}\left[\left(\beta-C_{1}(\tilde{\beta})\right)^{2}+\left(\beta-C_{2}(\tilde{\beta})\right)^{2}\right]-\gamma\left(\beta-C_{1}(\tilde{\beta})\right)\left(\beta-C_{2}(\tilde{\beta})\right)
$$

the gain of a firm with true cost parameter $\beta$ when it announces $\tilde{\beta}$ to the unique regulator. The firm will reveal truthfully its private information if

$$
\beta \in \arg \max _{\tilde{\beta}} U(\beta ; \tilde{\beta}) \text { or }\left\{\begin{array}{l}
\dot{U}(\beta)=-(1+\gamma)\left[e_{1}(\beta)+e_{2}(\beta)\right] \\
\dot{e}_{1}(\beta)+\dot{e}_{2}(\beta) \leq 2
\end{array}\right.
$$

\footnotetext{
${ }^{9}$ When the action taken by a regulator directly affects the welfare of the other regulator (not just through the rent of the firm), Martimort and Stole (1998) show that decentralization leads to multiple equilibria (under complete and asymmetric information). Hence, in this case decentralization yields different outcomes than centralization, even under complete information.

${ }^{10}$ This is due to the intrinsic common agency assumption. Had we assumed that the firm could decide to realize a project for only one country, the optimal efforts would not have been changed; however, each transfer would have been defined uniquely.

${ }^{11}$ See Laffont and Tirole (1993) for instance.

${ }^{12}$ See Gibbard (1973), Green and Laffont (1977) or Myerson (1979).
} 
where $U(\beta)$ is the rent of the firm with type $\beta$ when it announces the truth to the regulator.

The centralized regulator must still ensure that the firm is willing to participate to the regulatory process, or that the firm earns a greater rent than its outside opportunity.

As is usual, we rewrite the objective function of the regulator in terms of efforts and rent instead of costs and transfer. The program of the centralized regulator can then be stated as follows:

$$
\left\{\begin{array}{l}
\max _{\left\{U(.), e_{1}(.), e_{2}(.)\right\}} \mathbf{E}_{\beta}\left\{S_{1}+S_{2}-(1+\lambda)\left[2 \beta-e_{1}(\beta)-e_{2}(\beta)+\psi\left(e_{1}(\beta), e_{2}(\beta)\right)\right]-\lambda U(\beta)\right\} \\
\text { subject to } \forall \beta \in[\underline{\beta}, \bar{\beta}] \\
\dot{U}(\beta)=-(1+\gamma)\left[e_{1}(\beta)+e_{2}(\beta)\right] \\
\dot{e}_{1}(\beta)+\dot{e}_{2}(\beta) \leq 2 \\
U(\beta) \geq 0 .
\end{array}\right.
$$

We give the solution in the following proposition.

Proposition 3 Under asymmetric information and centralized regulation, the optimal levels of effort are symmetric and are given by

$$
e_{1}(\beta)=e_{2}(\beta)=e_{c}(\beta)=\frac{1}{1+\gamma}\left[1-(1+\gamma) \frac{\lambda}{1+\lambda} \frac{F(\beta)}{f(\beta)}\right] .
$$

Effort is distorted downwards, except for the most efficient firm. Indeed, because the rent decreases with the efficiency parameter, the effort provided by less efficient firms must be decreased in order to limit the rents of the more efficient ones. This is the standard trade-off between rent extraction and incentive to effort: on the one hand, for efficiency reasons the regulator would like to implement effort levels that are not too distorted with respect to their first-best levels; on the other hand, the higher the effort required from the firm, the larger the rent given up to the firm, and consequently the larger the social cost due to this rent. Also, all firms, except the most inefficient one, earn a positive rent. Asymmetric information forces the unique regulator to leave a positive, and socially costly, rent to the firm in order to obtain truthful revelation of the private information. 


\section{Decentralized regulation under asymmetric infor- mation}

We first start with a description of the way we solve this multiprincipal problem. The methodology is borrowed from Martimort and Stole (1998). Then we compute the optimal contracts.

The literature on common agency has exhibited many failures of a direct application of the Revelation Principle. Once it becomes impossible to rely on direct mechanisms to characterize the outcome of the common agency game, one has to consider indirect mechanisms. A priori, these mechanisms are based on very general (and untractable) spaces. However, Martimort and Stole (1998) have shown that there is no loss of generality in restricting regulator $P_{i}$ to use a non linear transfer based on the observable cost $C_{i}$ incurred by the firm on activity $i^{13}$. Otherwise stated, it is useless to consider a more complicated contract (that would include an extra-message sent by the firm).

Importantly, we know now that the optimal contract of a regulator for a given contract proposed to the firm by the other regulator belongs to this class of mechanisms. Also, from now on we will restrict ourselves to twice differentiable non linear deterministic $\operatorname{transfers}^{14}$.

\subsection{The problem of regulator $P_{1}$}

In this subsection, we characterize the best-response of the regulator in country 1 to any contract proposed by the other regulator. First, for any non linear transfer $t_{2}\left(C_{2}\right)$ offered by $P_{2}$ we can apply the Revelation Principle to find $P_{1}$ 's best-response. However, different contracts proposed by $P_{2}$ affect differently the firm's incentives to produce for $P_{1}$ and therefore $P_{1}$ 's best-response. Consequently let us define the firm's indirect utility function as

$$
\hat{U}^{1}\left(C_{1}, \beta\right)=\max _{C_{2}} \quad\left\{t_{2}\left(C_{2}\right)-\frac{1}{2}\left[\left(\beta-C_{1}\right)^{2}+\left(\beta-C_{2}\right)^{2}\right]-\gamma\left(\beta-C_{1}\right)\left(\beta-C_{2}\right)\right\} .
$$

This indirect utility function gives the maximal gain of a $\beta$-type firm (excluding the transfer received from regulator $P_{1}$ ) for a given cost $C_{1}$ on activity 1 when the firm

\footnotetext{
${ }^{13}$ They call this result the Taxation Principle. This result hinges on the quasi-linearity of the firm's utility function with respect to monetary transfers.

${ }^{14}$ This restriction is standard in the common agency literature.
} 
chooses optimally its cost level $C_{2}$ on activity 2 . Rewriting this function as $\hat{U}^{1}\left(\beta-e_{1}, \beta\right)$ we see that it determines the rate at which the firm must incur effort to compensate for a lie on $\beta$, and therefore its information rent. Hence, under decentralization there is an informational externality created by one regulator which affects the way the rival regulator must design his contract. For further reference, we denote by $C_{2}^{*}\left(C_{1}, \beta\right)$ the cost on activity 2 which satisfies the first-order condition ${ }^{15}$ associated with the previous problem, that is

$$
t_{2}^{\prime}\left(C_{2}^{*}\left(C_{1}, \beta\right)\right)+\beta-C_{2}^{*}\left(C_{1}, \beta\right)+\gamma\left(\beta-C_{1}\right)=0 .
$$

Given a contract offered to the firm by $P_{2}$, we can apply the Revelation Principle to find the implementable contracts from the point of view of $P_{1}$. A firm with type $\beta$ will reveal its private information if

$$
\beta \in \underset{\tilde{\beta}}{\arg \max } U(\tilde{\beta} ; \beta)=t_{1}(\tilde{\beta})+\hat{U}^{1}\left(C_{1}(\tilde{\beta}), \beta\right) .
$$

Local incentive compatibility implies ${ }^{16}$

$$
\left\{\begin{array}{l}
\dot{U}(\beta)=\hat{U}_{\beta}^{1}\left(C_{1}(\beta), \beta\right) \\
\dot{C}_{1}(\beta) \hat{U}_{1 \beta}^{1}\left(C_{1}(\beta), \beta\right) \geq 0
\end{array}\right.
$$

where $U(\beta)$ is now the rent of the firm in a truthful equilibrium. Immediate manipulations enable us to rewrite $P_{1}$ 's problem as

$$
\left\{\begin{array}{l}
\max _{\left\{U(.), C_{1}(.)\right\}} \mathbf{E}_{\beta}\left\{S_{1}-(1+\lambda)\left[C_{1}(\beta)-\hat{U}^{1}\left(C_{1}(\beta), \beta\right)\right]-\left(1+\lambda-\delta_{1}\right) U(\beta)\right\} \\
\text { subject to } \forall \beta \in[\beta, \bar{\beta}] \\
\dot{U}(\beta)=\hat{U}_{\beta}^{1}\left(C_{1}(\beta), \beta\right) \\
\dot{C}_{1}(\beta) \hat{U}_{1 \beta}^{1}\left(C_{1}(\beta), \beta\right) \geq 0 \\
U(\beta) \geq 0
\end{array}\right.
$$

\footnotetext{
${ }^{15}$ To consider the out of equilibrium behavior of the firm, the transfer $t_{2}\left(C_{2}\right)$ has to be extended for costs which may lie outside the set of equilibrium allocations in order that $C_{2}^{*}\left(C_{1}, \beta\right)$ be always defined by the first-order condition (1). See Martimort (1992) for the construction of such extensions.

${ }^{16}$ Subscripts on the indirect utility function denote without ambiguity partial derivatives.
} 
If the equivalent of the Spence-Mirrlees condition, $\hat{U}_{1 \beta}^{1}\left(C_{1}(\beta), \beta\right) \geq 0$, is satisfied, then the local second-order condition reduces to $\dot{C}_{1}(\beta) \geq 0$ and local incentive conditions are sufficient for global incentive compatibility. This condition cannot be postulated a priori as it depends endogenously on the contract proposed by the rival regulator. Hence, it must be checked ex post at the equilibrium.

Moreover, we have expressed the optimization behavior of the firm with respect to each regulator. It remains to check that it defines a global maximum for the firm (i.e. that the firm is effectively willing to accept simultaneously both contracts in equilibrium).

\subsection{The ambiguous effect of decentralization}

When regulators do not cooperate we obtain the following proposition. To obtain a symmetric equilibrium, we assume $\delta_{1}=\delta_{2}=\frac{1}{2}$.

Proposition 4 Under decentralization with asymmetric information the optimal profiles of effort in a symmetric equilibrium are characterized by

$$
e_{1}(\beta)=e_{2}(\beta)=e_{d}(\beta)=\frac{1}{1+\gamma}\left[1-(1+\gamma) \frac{\frac{1}{2}+\lambda}{1+\lambda} \frac{F(\beta)}{f(\beta)} \frac{1-\gamma+2 \gamma \dot{e}_{d}(\beta)}{1+\gamma \dot{e}_{d}(\beta)}\right]
$$

with initial condition $e_{d}(\underline{\beta})=e_{*}(\underline{\beta})$ and $e_{d}(\beta) \leq e_{*}(\beta)$ for all $\beta$.

- If efforts are strongly substitutable $\left(\gamma \geq \frac{1}{1+2 \lambda}\right)$ then all the optimality conditions are satisfied and, moreover, $e_{d}(\beta) \geq e_{c}(\beta)$ for all $\beta$; therefore the rent of the firm is larger under decentralization than under centralization.

- If efforts are weakly substitutable $\left(\gamma<\frac{1}{1+2 \lambda}\right)$ then the optimality conditions cannot be checked directly and $e_{d}(\beta)$ might be larger or smaller than $e_{c}(\beta)$.

To understand in depth the two effects at work, let us first consider the case of unrelated efforts (i.e. $\gamma=0$ ). In this situation, the multiprincipal aspect disappears as the contract offered by one regulator does not affect the choice of effort (or cost) by the firm for the other regulator and the problems of the regulators become separable (up to the participation constraint of the firm).

However, even in this case, decentralization is not equivalent to centralization for the following reason. Under centralization, the regulator fully internalizes the impact of his 
regulation on the rent of the firm that entirely goes to the consumers of both countries: one unit of rent left to the firm has a social cost of $(1+\lambda)-1=\lambda$.

Under decentralization, this is no longer the case. As a given regulator is only interested in the welfare of the consumers in his country, he does not internalize the effect of his regulation on the fraction of the rent that accrues to the shareholders of the other country. As a consequence, under decentralization, $P_{i}$ 's perceived cost of one unit of rent given up to the firm is $(1+\lambda)-\delta_{i}$ which is larger than the social evaluation of the firm's rent under centralization. We call this effect the shared-rent externality. ${ }^{17}$

Let alone, the shared-rent externality has a clear impact on the regulatory contracts offered to the firm under decentralization. Indeed, as the centralized regulator attaches more weight to the firm's rent than each decentralized regulator, the efforts under centralization tend to be higher than those under decentralization (as rent extraction is more important under decentralization because the firm's rent is more costly for each regulator). Hence, the larger the firm's rent the larger the distortion due to decentralization. Obviously, this externality is present whatever the degree of substitutability of efforts.

Notice also that the larger the shadow cost of public funds $\lambda$, the less important the shared-rent externality becomes as the discrepancy between the weight attached to the firm's rent under centralization and decentralization decreases (relatively to the weight of the consumers' surplus).

Finally, notice that this effect would have disappeared had we assumed that the shareholders were not in the countries where the projects are realized ${ }^{18}$.

Secondly, let us explain the effect of decentralization on the power of the incentive contracts when efforts are related (i.e. $\gamma \neq 0$ ). Under centralization, the unique regulator completely coordinates the choice of efforts and anticipates that a firm maximizing its profit will substitute one effort to the other in order to increase its rent.

Under decentralization, when regulator $P_{1}$ requires an effort from the firm he also anticipates, but cannot control for, that the firm will try to take advantage of the uncoordinated regulations by substituting one effort to the other. Then $P_{1}$ will require from the firm to exert more effort than under a centralized regulation. In equilibrium, these anticipations realize and indeed more effort is required by each regulator. Roughly speaking,

\footnotetext{
${ }^{17}$ Decentralization fails to internalize shared-rent externalities. It is an example of coordination failure due to decentralization.

${ }^{18}$ This assumption is often made in the multiprincipal literature.
} 
regulators are competing for the firm and this behavior leads to an increase in the power of the incentive contracts offered in equilibrium. This is the competition effect. ${ }^{19}$

Obviously, the more substitutable the efforts from the point of view of the firm are, the larger the competition effect is and the larger the distortion due to decentralization is.

When both effects are taken simultaneously into account, the total distortion due to decentralization is ambiguous as the two effects previously mentioned go in opposite directions. The shared-rent externality leads the decentralized regulators to offer lowerpowered contracts while the competition effect induces them to propose higher-powered incentive regulations.

As stated in the proposition, one can nonetheless show that when $\gamma \geq \frac{1}{1+2 \lambda}$, i.e., when efforts are sufficiently substitutable and/or the shadow cost of public funds is sufficiently large, decentralization always results in larger efforts than centralization: the competition effect dominates the shared-rent externality, and the firm earns a larger rent under decentralization. We give a surprising illustration of this in the next subsection.

Finally, the last part of the proposition is more technical and indicates that the verification of the optimality conditions becomes complex when the ranking of efforts is ambiguous. In the appendices, we show that these conditions are always satisfied in the uniform case. For this case efforts are linear ${ }^{20}$ in the efficiency parameter and take the same value for the most efficient firm. Comparing these efforts by computing the difference between their slopes, we obtain

$$
\dot{e}_{d}(\beta)-\dot{e}_{c}(\beta) \propto \gamma(1+4 \lambda)-1 \quad \text { (uniform case) }
$$

which illustrates our discussion: for large values of the substitutability index, decentralization leads to larger efforts than centralization (competition effect) whereas for low values of the shadow cost of public funds, the reverse always holds (shared-rent externality).

\footnotetext{
${ }^{19} \mathrm{It}$ is a second type of coordination failure due to decentralization.

${ }^{20}$ In general, the solutions are not linear and it could be possible that for some values of the efficiency parameter $e_{d}(\beta)$ be larger than $e_{c}(\beta)$ whereas for other values the reverse would hold.
} 


\subsection{The role of efforts allocation and the drift of regulatory contracts towards fixed-price contracts}

As explained earlier, the competition effect depends mainly on the substitutability of efforts at the firm's level. When efforts are sufficiently substitutable, then decentralization leads to too large efforts.

One can also show that an increase in the degree of substitutability locally increases the effort of the more efficient firms. The possibility to allocate easily its efforts on one activity or the other hardens the competition effect. Competition between regulatory authorities attains then its paroxysm when efforts are perfectly substitutable and in this case, we can even prove the following result.

Proposition 5 When efforts are perfectly substitutable $(\gamma=1)$ there exists an equilibrium in which both regulators offer a fixed-price contract to the firm ${ }^{21}$.

When $P_{2}$ offers a fixed-price contract to the firm, and when $\gamma=1$, we show in the appendices that $\hat{U}_{1 \beta}^{1}\left(C_{1}(\beta), \beta\right)$ is equal to 0 . This implies that the second-order condition for implementability is (weakly) satisfied; however, this also implies that regulator $P_{1}$ can no longer distort the effort he requires to limit the firm's rent, and cannot trade-off rent extraction and efficiency.

This is a striking illustration of the drift of the regulatory contract. Efforts are equal to the first-best efforts but the rent given up to the firm by the regulators becomes very large. Competition between regulatory authorities leads to large inefficiencies and prevent them from distorting their policy.

In the next section, we shall build on this insight.

\footnotetext{
${ }^{21}$ In the uniform case with perfectly substitutable efforts that we use in the next section there will be two candidate solutions to the differential equation characterizing the optimal effort under decentralization. However, for this case, the one that does not correspond to the fixed-price contract violates the implementability conditions. It is immediate to show that this is also the case for all the probability distributions with a linear hazard rate $\left(\frac{F(\beta)}{f(\beta)}=l(\beta-\underline{\beta}), l>0\right)$.
} 


\section{The choice of the regulatory structure under po- litical uncertainty}

Political economy has often challenged the view that the regulatory authority acts as a benevolent planner ${ }^{22}$. The goal of this section is to recognize that the authority in charge of the regulation in each country has a private agenda; we take the example of politicians who only seek to maximize their probability of being reelected ${ }^{23}$ and look at the impact of decentralization in such a setting. Another interpretation would be that the regulator(s) can be captured by some interest groups that try to distort the regulation in their own interest $^{24}$.

Let us assume now that in the two regions there is a random proportion of shareholders (resp. non shareholders) denoted by $\alpha_{i}$ (resp. $\left.1-\alpha_{i}\right) \in[0,1]$. The shareholders of the firm benefit from the rent of the firm while the non shareholders do not.

Before the value of $\alpha_{i}, i=1,2$, is known, the constitution decides which regulatory structure (centralization or decentralization) to set up. However, this choice has to take into account that the regulators in place will act in a distortive way. In our static framework, we model this divergence between the objective of the regulator(s) and the interests of all the citizens by recognizing that the regulator(s) only care(s) about the majority in place.

Under decentralization, if $\alpha_{i}>\frac{1}{2}$, then there will be a (local) shareholder majority in region $i$. In this case the objective of the regulator in region $i$ will take into account only the surplus of the shareholders in this region and the part of the rent of the firm that accrues to these shareholders. On the contrary, when $\alpha_{i}<\frac{1}{2}$ there will be a non shareholder majority and the regulator in place will only care about the surplus of the non shareholders. Accordingly, the objective function of the regulator of region $i$ under decentralization is given by ${ }^{25}$

$$
S W_{i, d}= \begin{cases}\alpha_{i}\left[S_{i}-(1+\lambda)\left(t_{i}+C_{i}\right)\right]+\frac{\alpha_{i}}{\alpha_{1}+\alpha_{2}} U & \text { if } \alpha_{i}>\frac{1}{2} \\ \left(1-\alpha_{i}\right)\left[S_{i}-(1+\lambda)\left(t_{i}+C_{i}\right)\right] & \text { if } \alpha_{i}<\frac{1}{2}\end{cases}
$$

\footnotetext{
${ }^{22}$ See Buchanan(1965), Noll (1983) and Olson (1963) among others.

${ }^{23}$ See Laffont (1996).

${ }^{24}$ See Stigler (1971) for example and Bardhan and Mookherjee (1999) for a discussion of decentralization in terms of relative captures of local and central government.
} 
Under centralization, the unique regulator cares only about the (national) majority over both regions. His objective function will be

$$
S W_{c}= \begin{cases}\sum_{i=1}^{2} \alpha_{i}\left[S_{i}-(1+\lambda)\left(t_{i}+C_{i}\right)\right]+U & \text { if } \alpha_{1}+\alpha_{2}>1, \\ \sum_{i=1}^{2}\left(1-\alpha_{i}\right)\left[S_{i}-(1+\lambda)\left(t_{i}+C_{i}\right)\right] & \text { if } \alpha_{1}+\alpha_{2}<1 .\end{cases}
$$

To summarize, the different majorities have different stakes in the information rent of the firm, and the regulators have private agendas depending on the majority in power. Under centralization, the regulator will bias his regulation to favor the majority over both regions; on the contrary, decentralization makes the regulators compete against each other and act only in favor of the local majority. Notice also that both types of majority only differ in their treatment of the firm's rent.

The performances of these different regulatory structures have to be compared with respect to the utilitarian criterion defined as usual by

$$
S W^{u}=\sum_{i=1}^{2}\left\{S_{i}-(1+\lambda)\left(t_{i}+C_{i}\right)\right\}+U .
$$

In the following, we shall determine the profiles of effort implemented by each constitution. Observe that under a shareholder majority the rent of the firm is overvalued while under a non shareholder majority the rent of the firm is undervalued with respect to the utilitarian criterion.

For expositional purposes, we restrict attention to the uniform case, with $\alpha_{1}=\alpha_{2}=\alpha$ and with efforts perfectly substitutable for the firm $(\gamma=1)^{26}$. We also assume that under a shareholder majority $(1+\lambda) \alpha-\frac{1}{2}>(1+\lambda) \alpha-1>0$ for rent extraction to be desirable under decentralization and centralization.

\subsection{The profiles of effort}

We can adapt our previous computations since only the weight of the firm's rent is changed in the objective function of the regulators. The optimal efforts are given in the next

\footnotetext{
${ }^{25}$ Letter 'd' (resp. 'c') stands for decentralization (resp. centralization).

${ }^{26}$ In a previous draft, we did not restrict ourselves to the case $\gamma=1$. One can show that our insights carry over (qualitatively) to the situations in which efforts are sufficiently substitutable. When the proportion of shareholders in both regions can be different, decentralization may lead to non monotonic profiles of efforts, but once again our argument could be extended to such cases.
} 
proposition.

Proposition 6 The optimal profiles of effort are given by:

- Under centralization $e_{c}(\beta)=\frac{1}{2}\left[1-2 r_{c} \beta\right]$ where $r_{c}=1$ under a non shareholder majority and $r_{c}=\frac{(1+\lambda) \alpha-1}{(1+\lambda) \alpha}$ under a shareholder majority.

- Under decentralization $e_{d}(\beta)=\frac{1}{2}$ whatever the majority.

- With the utilitarian criterion $e_{u}(\beta)=\frac{1}{2}\left[1-2 \frac{\lambda}{1+\lambda} \beta\right]$.

This proposition calls for some comments. Under centralization, the optimal profile of effort fluctuates with the majority in place. Under a shareholder majority the effort is larger than the one corresponding to the utilitarian criterion as the regulator in place accounts for the share of the firm's rent that goes to the actual majority ${ }^{27}$. On the contrary, under a non shareholder majority effort is downward distorted with respect to its utilitarian level.

The decentralization of the regulatory powers leads to the striking result that the implemented efforts become insensitive to the majority in place. As explained earlier, this result comes from the perfect substitutability of the efforts provided by the firms which exacerbates the tension between the non cooperative regulators. This competition between institutions finally ends up with the regulators being forced to offer fixed-price contracts without the possibility to match the will of the majority in place with the effort required from the firm: decentralization leads to uniform policies with respect to the political majority.

When the non shareholders have the majority, the effort is too low under centralization and too high under decentralization. However, immediate computations show that decentralization distorts less the effort than centralization when the shadow cost of public funds is small $(\lambda<1)$. Hence, if the efficiency consideration is more important than the rent extraction one, it is intuitive that under a non shareholder majority decentralization is preferred. This will be confirmed in the next subsection in which we perform some welfare comparisons.

\footnotetext{
${ }^{27}$ More precisely, this is due to the fact that the centralized regulator only cares about the shareholders which implies that the relative weight of the firm's rent (with respect to the weight attached to the net consumers' surplus) is larger under centralization than with the utilitarian criterion.
} 
Under a shareholder majority, both constitutions lead to too high effort levels. However, immediate computations show that centralization distorts less the effort than centralization under the assumption $(1+\lambda) \alpha>1$. Notice that when the proportion of shareholders is large (i.e. $\alpha$ close to 1 ) then the objective of the centralized regulator almost coincides with the utilitarian criterion, and the loss entailed by decentralization is large. In a similar way, the larger the social cost of public funds is, the more desirable centralization is.

Hence, the comparison between centralization and decentralization is ambiguous. On the one hand, centralization enables to implement efforts that limit the rent earned by the firm while decentralization always leaves too large rent to the firm. On the other hand, centralization is sensitive to the majority in place and leads to fluctuations in the levels of effort that favor the members of the majority. The comparison between centralization and decentralization hinges simultaneously on the proportion of shareholders/non shareholders and on the shadow cost of public funds, which gives a measure of the social cost of the firm's rent. Effort levels are represented in Figure 1.

$$
\text { Insert Figure } 1 \text { here }
$$

\subsection{Welfare analysis}

To assess the performances of centralization and decentralization, we must then compare the expected welfares of both countries under the different constitutions. For a given majority with size $\alpha$ that implements the profile of efforts $e(\beta, \alpha)$, the expected social welfare is given by

$\mathbf{E}_{\beta}\left\{S W^{u}(e(\beta, \alpha))\right\}=\int_{\underline{\beta}}^{\bar{\beta}}\left\{S_{1}+S_{2}-(1+\lambda)[\psi(e(\beta, \alpha))+2(\beta-e(\beta, \alpha))]-\lambda U(\beta)\right\} d F(\beta)$.

Whatever the regulatory structure, the rent of the firm in a symmetric equilibrium is given by

$$
\dot{U}(\beta)=-4 e(\beta, \alpha)
$$


which gives (after an integration by parts) in the uniform case

$\mathbf{E}_{\beta}\left\{S W^{u}(e(\beta, \alpha))\right\}=S_{1}+S_{2}-(1+\lambda)-2 \int_{0}^{1}\{(1+\lambda) e(\beta, \alpha)[e(\beta, \alpha)-1]+2 \lambda \beta e(\beta, \alpha)\} d \beta$.

Depending on the majority in place, the expected welfare under centralization is given $\mathrm{by}^{28}$

$\begin{cases}S W_{c, s}^{u} & =S_{1}+S_{2}-(1+\lambda)+\frac{4(2 \alpha-1)-\alpha^{2}\left(1-\lambda^{2}\right)}{6 \alpha^{2}(1+\lambda)} \text { with a shareholder majority } \\ S W_{c, n s}^{u} & =S_{1}+S_{2}-(1+\lambda)-\frac{1-\lambda}{6} \text { with a non shareholder majority }\end{cases}$

Under decentralization, because efforts are not dependent on the majority in place, the expected welfare of both countries is

$$
S W_{d, s}^{u}=S W_{d, n s}^{u}=S_{1}+S_{2}-(1+\lambda)+\frac{1-\lambda}{2} \text { whatever the majority. }
$$

Then, the difference between the welfare under centralization and the one under decentralization is

$$
\left\{\begin{array}{l}
S W_{d, s}^{u}-S W_{c, s}^{u}=\frac{2}{3} \frac{(1-\alpha)^{2}-\alpha^{2} \lambda^{2}}{\alpha^{2}(1+\lambda)} \text { under a shareholder majority, } \quad(\alpha>1 / 2) \\
S W_{d, n s}^{u}-S W_{c, n s}^{u}=\frac{2}{3}(1-\lambda) \text { under a non shareholder majority. } \quad(\alpha<1 / 2)
\end{array}\right.
$$

We assume that the probabilities to have a shareholder majority and a non shareholder majority are the same (equal to $\frac{1}{2}$ ). This enables us to state the following proposition. ${ }^{29}$

Proposition 7 If the shadow cost of public funds is large $(\lambda \geq 1)$ then centralization is preferred to decentralization. On the contrary, when the shadow cost of public funds is low $(\lambda \leq 1 / \sqrt{2})$ then decentralization is preferred to centralization.

For intermediate values of the shadow cost of public funds $(1 / \sqrt{2}<\lambda<1)$, then decentralization (resp. centralization) is preferred to centralization (resp. decentralization) when the shareholder majority is weak (resp. large)..$^{30}$

\footnotetext{
${ }^{28}$ Letter 's' (resp. 'ns') stands for shareholder (resp. non shareholder) majority.

${ }^{29}$ Under a shareholder majority, the assumption $(1+\lambda) \alpha>1$ implies that $(1-\alpha)^{2}-\alpha^{2} \lambda^{2}<0$.

${ }^{30}$ The size of the non shareholder majority does not affect the effort levels.
} 
This proposition confirms the intuitions derived from the comparison of the effort levels. Indeed, when the shadow cost of public funds is large, then the rent left to the firm has a large social cost. Moreover, under decentralization the competition between regulatory authorities provides the firm with excessive rent. These two effects work in favor of centralization and give the rationale for the first part of the proposition.

When the shadow cost of public funds is small then decentralization is preferred under a non shareholder majority. Moreover, even if centralization is preferred under a shareholder majority, the loss entailed by decentralization tends to be small. Hence, the former effect more than offsets the latter, and decentralization is preferred.

For intermediate values, the trade-off also depends on the size of the majority in power. The drawback of centralization is that the unique regulator only cares about the majority. Hence, when the size of the majority is small, the proportion of consumers disadvantaged by the centralized regulator tends to be relatively large and decentralization becomes the preferred constitution even though it provides the firm with too much rent (which has a low social cost if $\lambda$ is not too large). Decentralization serves to limit the discretionary power of the regulators.

\section{Conclusion}

We have compared the performances of centralization and decentralization of the regulatory powers using the new regulatory economics and without appealing to any informational advantage under decentralization or externalities between countries.

In this setting, the benefit of centralization of the regulatory power at a supranational level is to coordinate the regulations and to take into account the informational externality created by the link between both activities at the firm's level.

Decentralization is plagued by two opposite distortions. The first relates to the informational externality which translates into a competition effect when efforts are substitutes. The second comes from the fact that a regulator does not internalize the impact of his regulation and the fraction of the firm's rent that accrues to the shareholders of the other country.

If efforts were complements instead of substitutes then the competition effect would be reversed ${ }^{31}$ : a regulator would free-ride on the incentives provided to the firm by the other regulator and this would lead to too low-powered incentive contracts in equilib- 
rium. Moreover, this under-provision of incentives would be reinforced by the shared-rent externality.

Then, we introduced a bias in the objective of the regulator. Using the random majority model, in which the regulator only cares about the majority in place, we show that decentralization could perform better than centralization. Indeed, decentralizing the decision power modifies the political rules of the game played by the decision-makers and creates a competition between regulators. In our setting, this competition eliminates the negative discretionary power of the regulators at the cost of providing the firm with excessive rent. This effect would be still present if efforts were complements as decentralization would still reduce the distortion under a shareholder majority. In the same vein, introducing a degree of 'competitiveness' (through, say, an unregulated fringe in each country producing an imperfectly differentiated product) in our model would just modify the equilibrium rent of the regulated firm but would not alter qualitatively our conclusions.

\footnotetext{
${ }^{31}$ With complements, there exists a continuum of equilibria that always lead to lower effort than centralization.
} 


\section{References}

Auriol, E. and J.J. Laffont, 1992, "Regulation by Duopoly", Journal of Economics and Management Strategy, 1, 503-533.

Bardhan, P. and D. Mookherjee, 1999, "Relative Capture of Local and Central Governments: An Essay in the Political Economy of Decentralization", University of California at Berkeley.

Bernheim, D. and M. Whinston, 1986, "Menu Auctions, Resource Allocations, and Economic Influence", Quarterly Journal of Economics, 101: 1-31.

Buchanan, J., 1965, "An Economic Theory of Clubs", Econometrica, 33: 1-14.

Caillaud B., B. Jullien and P. Picard, 1996, "National vs European Incentive Policies: Bargaining, Information and Coordination", European Economic Review, 40: 91-112.

Dewatripont, M. and J. Tirole, 1999, "Advocates", Journal of Political Economy, 107, 1-39.

Gibbard, A., 1973, "Manipulation for Voting Schemes", Econometrica, 41: 587-601.

Gilbert, G. and P. Picard, 1996, "Incentives and Optimal Size of Local Juridictions" European Economic Review, 40, 19-42.

Green, J. and J.J. Laffont, 1977, "Characterization of Satisfactory Mechanisms for the Revelation of Preferences for Public Goods", Econometrica, 45, 42735.

Hart, O. and J. Moore, 1999, "On the Design of Hierarchies: Coordination versus Specialization", mimeo.

Klibanoff, P. and M. Poitevin, 1997, "A Theory of (de)centralisation", mimeo CIRANO, Universit de Montral.

Laffont, J.J., 1996, "Industrial Policy and Politics", International Journal of Industrial Organisation, 14: 1-27.

Laffont, J.J. and D. Martimort, 1998, "Collusion and Delegation", Rand Journal of Economics, 29: 280-305. 
Laffont, J.J. and J. Tirole, 1986, "Using Cost Observation to Regulate Firms", Journal of Political Economy, 94, 614-641.

Laffont, J.J. and J. Tirole, 1993, A Theory of Incentives in Procurement and Regulation, M.I.T. Press, Cambridge, Ma.

Laffont, J.J. and W. Zantman, 1999, "Information Acquisition, Political Game and the Delegation of Authority", Working Paper GREMAQ, Universit de Toulouse 1.

Martimort, D., 1992, "Multi-principaux avec anti-sélection", Annales d'Economie et Statistiques, 28: 1-38.

Martimort, D., 1999, "Renegotiation Design with Multiple Regulators", Journal of Economic Theory,

Martimort, D. and L. Stole, 1998, "Contractual Externalities and Common Agency Equilibria", mimeo.

Myerson, R., 1979, "Incentive Compatibility and the Bargaining Problem", Econometrica, vol. 47, 61-73.

Noll, R., 1983, "The Political Foundations of Regulatory Politics", Journal of Institutional and Theoretical Economics, 139: 377-404.

Olsen, T. and G. Torsvick, 1993, "The Ratchet Effect in Common Agency: Implication for Regulation and Privatization", Journal of Law, Economics and Organization, 9, 136-158.

Olson, M., 1965, The Logic of Collective Actions, Harvard University Press, Cambridge, Ma.

Sah, R. and J. Stiglitz, 1986, "The Architecture of Economic Systems: Hierarchies and Polyarchies", American Economic Review, 76, 716-727.

Seabright, P., 1996, "Accountability and Decentralization in Government: An Incomplete Contracts Model", European Economic Review, 40: 61-89.

Stigler, G., 1971, "The Economic Theory of Regulation", Bell Journal of Economics, 2:3-21. 


\section{Appendices}

\subsection{Complete information}

Under centralization, because the rent is socially costly, the regulator sets $U=0$. Then replacing the value of the transfer in the objective function and optimizing with respect to efforts we obtain the first-best efforts.

Under decentralization the same methodology can be applied directly.

\subsection{Centralized regulation under asymmetric information}

As the rent is (strictly) decreasing in the efficiency parameter and because the rent is socially costly, the participation constraint amounts to $U(\bar{\beta})=0$. The Hamiltonian associated with the corresponding optimal control problem is

$$
\begin{aligned}
H=f(\beta) & {\left[S_{1}+S_{2}-(1+\lambda)\left(2 \beta-\left(e_{1}(\beta)+e_{2}(\beta)\right)\right.\right.} \\
+ & \left.\left.\frac{1}{2}\left(e_{1}(\beta)^{2}+e_{2}(\beta)^{2}\right)+\gamma e_{1}(\beta) e_{2}(\beta)\right)-\lambda U(\beta)\right]-\eta(\beta)(1+\gamma)\left[e_{1}(\beta)+e_{2}(\beta)\right] .
\end{aligned}
$$

Applying the Maximum Principle we get $\dot{\eta}(\beta)=\lambda f(\beta)$. Because there is no transversality condition at $\underline{\beta}, \eta(\underline{\beta})=0$ and we obtain $\eta(\beta)=\lambda F(\beta)$. Then optimizing with respect to $e_{i}(\beta)$ we obtain the optimal profiles of effort. Finally, under the monotone hazard rate assumption, $\frac{d}{d \beta} \frac{F(\beta)}{f(\beta)} \geq 0$, the second-order condition for implementability is satisfied.

\subsection{Decentralized regulation under asymmetric information}

\subsubsection{Preliminary results}

Immediate computations yield

- $\hat{U}_{1}^{1}\left(C_{1}, \beta\right)=\left(\beta-C_{1}\right)+\gamma\left(\beta-C_{2}^{*}\left(C_{1}, \beta\right)\right)$,

- $\hat{U}_{\beta}^{1}\left(C_{1}, \beta\right)=-(1+\gamma)\left(\beta-C_{1}+\beta-C_{2}^{*}\left(C_{1}, \beta\right)\right)$,

- $\hat{U}_{1 \beta}^{1}\left(C_{1}, \beta\right)=(1+\gamma)\left(1+\frac{\partial C_{2}^{*}\left(C_{1}, \beta\right)}{\partial C_{1}}\right)$,

where $C_{2}^{*}\left(C_{1}, \beta\right)$ is defined by the first-order condition (1) associated with the indirect utility function of the firm vis à vis regulator $P_{2}$. 
Differentiating this condition with respect to $C_{1}$ we obtain

$$
\left[t_{2}^{\prime \prime}\left(C_{2}^{*}\left(C_{1}(\beta), \beta\right)\right)-1\right] \frac{\partial C_{2}^{*}\left(C_{1}(\beta), \beta\right)}{\partial C_{1}}=\gamma .
$$

In equilibrium, $C_{2}^{*}\left(C_{1}(\beta), \beta\right)=C_{2}(\beta)$. Substituting in (1) and differentiating with respect to $\beta$ we also get

$$
\left[t_{2}^{\prime \prime}\left(C_{2}(\beta)\right)-1\right] \dot{C}_{2}(\beta)=-1-\gamma\left(1-\dot{C}_{1}(\beta)\right)
$$

Using (2) and (3), we obtain

$$
\hat{U}_{1 \beta}^{1}\left(C_{1}(\beta), \beta\right)=(1+\gamma) \frac{1+\gamma-\gamma\left(\dot{C}_{1}(\beta)+\dot{C}_{2}(\beta)\right)}{1+\gamma\left(1-\dot{C}_{1}(\beta)\right)} .
$$

\subsubsection{The symmetric equilibrium}

The optimal schedules of effort $\operatorname{As}_{U^{1}}^{1}\left(C_{1}, \beta\right)<0$ and because the rent is socially costly, the participation constraint amounts to $U(\bar{\beta})=0$. The Hamiltonian associated with the problem of regulator $P_{1}$ is

$$
f(\beta)\left[S_{1}-(1+\lambda)\left(C_{1}(\beta)-\hat{U}^{1}\left(C_{1}(\beta), \beta\right)\right)-\left(\frac{1}{2}+\lambda\right) U(\beta)\right]+\eta(\beta) \hat{U}_{\beta}^{1}\left(C_{1}(\beta), \beta\right) .
$$

Applying the Maximum Principle and using the fact that there is no transversality condition at $\underline{\beta}(\eta(\underline{\beta})=0)$, we obtain $\eta(\beta)=\left(\frac{1}{2}+\lambda\right) F(\beta)$. Finally, optimizing with respect to $C_{1}(\beta)$, considering a symmetric equilibrium and rearranging terms we obtain the optimal effort profiles.

Behavior of the solution in the neighborhood of $\beta$ In order to compare the efforts under centralization and decentralization, we must first linearize the solution to the differential equation in the neighborhood of $\underline{\beta}$. We have

$$
\dot{e}_{d}(\beta)=-\frac{1}{\gamma} \frac{(1+\gamma) e_{d}(\beta)-1+(1+\gamma)(1-\gamma) \frac{F(\beta)}{f(\beta)} \frac{\frac{1}{2}+\lambda}{1+\lambda}}{(1+\gamma) e_{d}(\beta)-1+2(1+\gamma) \frac{F(\beta)}{f(\beta)} \frac{\frac{1}{2}+\lambda}{1+\lambda}}
$$

Let us use the following notations: $X=e_{d}(\beta)-e_{d}(\underline{\beta})$ and $Y=\beta-\underline{\beta}$. Immediate computations show that (4) can be rewritten as

$$
\frac{d X}{d Y}=-\frac{1}{\gamma} \frac{X+(1-\gamma) \frac{\frac{1}{2}+\lambda}{1+\lambda} Y}{X+2 \frac{\frac{1}{2}+\lambda}{1+\lambda} Y}
$$


Looking for a solution of the form $X=t Y$, we must solve the following equation: $\gamma t^{2}+\left(2 \frac{\frac{1}{2}+\lambda}{1+\lambda} \gamma+1\right) t+(1-\gamma) \frac{\frac{1}{2}+\lambda}{1+\lambda}=0$. The two roots are given by

$$
\underline{t}_{d}=-\frac{1+2 \gamma \frac{\frac{1}{2}+\lambda}{1+\lambda}+\sqrt{\Delta}}{2 \gamma} \text { and } \bar{t}_{d}=\frac{-1-2 \gamma \frac{\frac{1}{2}+\lambda}{1+\lambda}+\sqrt{\Delta}}{2 \gamma} .
$$

where $\Delta=1+4 \gamma^{2} \frac{\frac{1}{2}+\lambda}{1+\lambda}\left(1+\frac{\frac{1}{2}+\lambda}{1+\lambda}\right)>0$ is the discriminant. It is immediate to show that

- $\underline{t}_{d}$ does not satisfy the optimality conditions of the firm's maximization problem given by (5),

- $\bar{t}_{d}<\dot{e}_{*}(\underline{\beta})$,

- $\bar{t}_{d}>\dot{e}_{c}(\underline{\beta})=-\frac{\lambda}{1+\lambda} \Leftrightarrow \gamma>\frac{1}{1+4 \lambda}$.

Comparative statics Let us consider the effect of an increase in the substitutability index $\gamma$ on the optimal effort under non cooperative regulations. In the neighborhood of $\underline{\beta}$ immediate computations yield $S g\left(\frac{d \bar{t}}{d \gamma}\right)=S g\left(1-\frac{1}{\sqrt{\Delta}}\right)>0$. Hence efforts increase locally, and globally in the uniform case, around $\underline{\beta}$ when $\gamma$ increases.

Let us now prove that $e_{d}(\beta) \leq e_{*}(\beta)$. First consider $\hat{\beta}$ such that $e_{d}(\hat{\beta})=e_{*}(\hat{\beta})$. At $\hat{\beta}$ we have $\dot{e}_{d}(\hat{\beta})=-\frac{1-\gamma}{2 \gamma} \leq \dot{e}_{*}(\hat{\beta})=0$. Hence, for $\beta \in(\hat{\beta}-\epsilon, \hat{\beta})$ we have $e_{d}(\beta)>e_{*}(\beta)$, a contradiction.

Let us find the conditions such that $e_{d}(\beta) \geq e_{c}(\beta) \forall \beta$. Consider $\hat{\beta}$ such that $e_{d}(\hat{\beta})=$ $e_{c}(\hat{\beta})$. Equation (4) gives

$$
\dot{e}_{d}(\hat{\beta})=\frac{\gamma \lambda-\frac{1}{2}(1-\gamma)}{\gamma(1+\lambda)} .
$$

Now assume that $\gamma \lambda-\frac{1}{2}(1-\gamma) \geq 0$ or $\gamma \geq \frac{1}{1+2 \lambda}$. Then $\dot{e}_{d}(\hat{\beta}) \geq 0$ while $\dot{e}_{c}(\hat{\beta}) \leq 0$ which in turn implies that $\forall \beta \in(\hat{\beta}-\epsilon, \hat{\beta}), e_{d}(\beta)<e_{c}(\beta)$. However, this contradicts the fact that if $\gamma \geq \frac{1}{1+2 \lambda} \geq \frac{1}{1+4 \lambda}$ then $\bar{t}_{d}>\dot{e}_{c}(\underline{\beta})$. Note finally that this a sufficient condition only.

The implementability conditions Let us check that the indirect utility function satisfies the Spence-Mirrlees property at the equilibrium. We have $\hat{U}_{1 \beta}^{1}\left(C_{1}(\beta), \beta\right)=(1+$ $\gamma)\left(1+\frac{\partial C_{2}^{*}\left(C_{1}(\beta), \beta\right)}{\partial C_{1}}\right)=(1+\gamma) \frac{1-\gamma+2 \gamma \dot{e}_{d}(\beta)}{1+\gamma \dot{e}_{d}(\beta)}=-(1+\gamma) \frac{(1+\gamma) e_{d}(\beta)-1}{(1+\gamma) \frac{F(\beta)}{f(\beta)} \frac{1}{2}+\lambda} \geq 0$ because $e_{d}(\beta) \leq e_{*}(\beta)$ $\forall \beta$. 
We check now for the monotonicity of the cost profile:

$$
\dot{C}_{d}(\beta) \geq 0 \Leftrightarrow 1-\dot{e}_{d}(\beta) \geq 0 \Leftrightarrow \frac{1+\gamma}{\gamma} \frac{(1+\gamma) e_{d}(\beta)-1+(1+\gamma) \frac{F(\beta)}{f(\beta)} \frac{\frac{1}{2}+\lambda}{1+\lambda}}{(1+\gamma) e_{d}(\beta)-1+2(1+\gamma) \frac{F(\beta)}{f(\beta)} \frac{\frac{1}{2}+\lambda}{1+\lambda}} \geq 0
$$

which is satisfied if $\gamma \geq \frac{1}{1+2 \lambda}$, since we have shown that under this condition $e_{d}(\beta) \geq e_{c}(\beta)$.

The optimality conditions for the firm We also must check that the problem of the firm is globally concave, or that the Hessian associated with the following maximization problem

$\max _{C_{1}(\beta), C_{2}(\beta)}\left\{t_{1}\left(C_{1}(\beta)\right)+t_{2}\left(C_{2}(\beta)\right)-\frac{1}{2}\left[\left(\beta-C_{1}(\beta)\right)^{2}+\left(\beta-C_{2}(\beta)\right)^{2}\right]-\gamma\left(\beta-C_{1}(\beta)\right)\left(\beta-C_{2}(\beta)\right)\right\}$

is definite semi-negative at the equilibrium. This is equivalent to the following two conditions

$$
\left\{\begin{array} { l l } 
{ t ^ { \prime \prime } ( C ( \beta ) ) - 1 } & { \leq 0 } \\
{ ( t ^ { \prime \prime } ( C ( \beta ) ) - 1 ) ^ { 2 } - \gamma ^ { 2 } } & { \geq 0 }
\end{array} \Leftrightarrow \left\{\begin{array}{ll}
1+\gamma \dot{e}_{d}(\beta) & \geq 0 \\
1-\gamma+2 \gamma \dot{e}_{d}(\beta) & \geq 0
\end{array}\right.\right.
$$

We have

$$
1-\gamma+2 \gamma \dot{e}_{d}(\beta)=-(1+\gamma) \frac{(1+\gamma) e_{d}(\beta)-1}{(1+\gamma) e_{d}(\beta)-1+2(1+\gamma) \frac{F(\beta)}{f(\beta)} \frac{\frac{1}{2}+\lambda}{1+\lambda}}
$$

which is positive since we have shown that $e_{*}(\beta) \geq e_{d}(\beta)$.

Simple computations yield

$$
1+\gamma \dot{e}_{d}(\beta)=\frac{(1+\gamma) \frac{F(\beta)}{f(\beta)} \frac{\frac{1}{2}+\lambda}{1+\lambda}}{(1+\gamma) e_{d}(\beta)-1+2(1+\gamma) \frac{F(\beta)}{f(\beta)} \frac{\frac{1}{2}+\lambda}{1+\lambda}}
$$

which is positive under the assumption $\gamma \geq \frac{1}{1+2 \lambda}$ since we have shown that in this case $e_{d}(\beta) \geq e_{c}(\beta)$.

\subsubsection{Solution in the uniform case}

The methodology used to compute the solution under decentralization in the uniform case is explained in the general case in section 8.4.1. It suffices to take $r_{d}=\frac{\frac{1}{2}+\lambda}{1+\lambda}$ in equations (6). The comparisons with the solution under centralization is immediate and left to the reader. 


\subsubsection{The fixed-price contract equilibrium}

Assume that $R_{2}$ offers a fixed-price contract to the firm and that $\gamma=1$. Then $\frac{\partial C_{2}^{*}\left(C_{1}, \beta\right)}{\partial C_{1}}=$ -1 implying $\hat{U}_{1 \beta}^{1}\left(C_{1}, \beta\right)=0$ and $e_{d}(\beta)=e_{*}(\beta)$. This profile of efforts can be implemented with a fixed-price contract.

\subsection{The choice of the regulatory structure under political un- certainty}

\subsubsection{Decentralization}

For region $i$ with a majority of $\delta_{i}$ the social welfare function of the local regulator can be rewritten as follows:

$$
S W_{i}=\delta_{i}\left[S_{i}-(1+\lambda)\left(C_{i}(\beta)-\hat{U}^{i}\left(C_{i}(\beta), \beta\right)\right)\right]-\left[(1+\lambda) \delta_{i}-\frac{\alpha_{i}}{\alpha_{1}+\alpha_{2}} \mathcal{I}_{\left\{\alpha_{i}>\frac{1}{2}\right\}}\right] U(\beta) .
$$

where $\mathcal{I}$ is the indicator function. Define $r_{d, i}=\frac{(1+\lambda) \delta_{i}-\frac{\alpha_{i}}{\alpha_{1}+\alpha_{2}} \mathcal{I}_{\left\{\alpha_{i}>\frac{1}{2}\right\}}}{(1+\lambda) \delta_{i}}$ and $\delta_{i}=\alpha_{i}$ if $\alpha_{i}>\frac{1}{2}$ or $\delta_{i}=1-\alpha_{i}$ if $\alpha_{i}<\frac{1}{2}$. Then, up to coefficient of the rent $U(\beta)$ in the social welfare function, the computations of the optimal profiles of efforts are similar. The implementability conditions are unchanged.

Lemma 1 With decentralization, the optimal profiles of effort under asymmetric information are characterized by

$$
\left\{\begin{array}{l}
e_{1}(\beta)+\gamma e_{2}(\beta)=1-r_{d, i}(1+\gamma) \frac{F(\beta)}{f(\beta)} \frac{1-\gamma+\gamma\left[\dot{e}_{1}(\beta)+\dot{e}_{2}(\beta)\right]}{1+\gamma \dot{e}_{1}(\beta)} \\
\gamma e_{1}(\beta)+e_{2}(\beta)=1-r_{d, i}(1+\gamma) \frac{F(\beta)}{f(\beta)} \frac{1-\gamma+\gamma\left[\dot{e}_{1}(\beta)+\dot{e}_{2}(\beta)\right]}{1+\gamma \dot{e}_{2}(\beta)}
\end{array}\right.
$$

with the initial conditions $e_{1}(\underline{\beta})=e_{2}(\underline{\beta})=e_{*}(\underline{\beta})$.

In the uniform case, when $\alpha_{1}=\alpha_{2}=\alpha$ then $r_{d, 1}=r_{d, 2}=r_{d}$. When assume that $(1+\lambda) \alpha>\frac{1}{2}$ for rent extraction to be desirable under a shareholder majority. We will look for linear and symmetric solutions of the form $e_{i}=a \beta+b$. Differentiating the optimality conditions, we obtain the following condition

$$
a=-r_{d} \frac{1-\gamma+2 \gamma a}{1+\gamma a}
$$


Solving (6) yields two candidate solutions. One can then show that one solution always fails to satisfy the optimality conditions of the firm's maximization problem (5) and can then be discarded from the analysis. We end up with

$$
a=\frac{-1-2 \gamma r_{d}+\sqrt{\Delta}}{2 \gamma},
$$

where $\Delta=1+4 \gamma^{2} r_{d}\left(r_{d}+1\right)>0$. We deduce then that $b=\frac{1}{1+\gamma}$.

Now we check that $1+\gamma a \geq 0$. If $1-2 \gamma r_{d} \geq 0$ then this condition is automatically satisfied. Otherwise this condition can be rewritten as $\gamma(1+\gamma) r_{d} \geq 0$ which obviously holds.

Now we must check that $1-\gamma+2 \gamma a \geq 0$. This condition is equivalent to $1-\gamma^{2} \geq 0$ which obviously holds.

Now we check that $\dot{C}(\beta) \geq 0$ or equivalently $a \leq 1$. This amounts to $\gamma(1+\gamma)\left(1+r_{d}\right) \geq$ 0 which obviously holds.

Finally, when $\gamma=1$, one can check immediately that the solutions are $a=0$ or $a=-1-2 r_{d}$. The last solution does not satisfy the optimality conditions of the firm's maximization problem (5).

\subsubsection{Centralization}

In the uniform case, with $\alpha_{1}=\alpha_{2}=\alpha$ and $(1+\lambda) \alpha>1$ for rent extraction to be desirable under a shareholder majority, immediate computations (adapted from section 8.2) show that the optimal profiles of effort are given by

$$
e_{1}(\beta)=e_{2}(\beta)=e_{c}(\beta)=\frac{1}{1+\gamma}\left[1-(1+\gamma) r_{c} \beta\right]
$$

where $r_{c}=\frac{(1+\lambda) \alpha-1}{(1+\lambda) \alpha}$ under a shareholder majority and $r_{c}=1$ under a nonshareholder majority.

\subsubsection{Expected welfares comparison}

Notice first that the proportion of shareholders appears only under a shareholder majority. With a slight abuse of notations, $\alpha$ represents now the proportion of shareholders under a shareholder majority (i.e. $\alpha>\frac{1}{2}$ ).

If $\lambda \geq 1$ then centralization is preferred whatever the majority. 
Assume now that $\lambda<1$. If both types of majority have the same probabilities $\left(\frac{1}{2}\right)$, then the difference between the expected welfare under decentralization and the expected welfare under centralization is proportional to

$$
P(\alpha)=2 \alpha^{2}\left(1-\lambda^{2}\right)-2 \alpha+1 .
$$

We must have $(1+\lambda) \alpha>1$ or $\lambda>\frac{1-\alpha}{\alpha}$, with $\alpha>1 / 2$.

The discriminant associated to $P(\alpha)$ is $4\left(2 \lambda^{2}-1\right)$. Consequently, if $\lambda^{2}<1 / 2$ then the discriminant is negative and $P(\alpha)>0$ for all values of $\alpha$ and $\lambda$ (as $1-\lambda^{2}>0$ by assumption).

Assume now that $\lambda^{2}>1 / 2$. The largest of the two roots associated to $P$ is

$$
\frac{1+\sqrt{2 \lambda^{2}-1}}{2\left(1-\lambda^{2}\right)}
$$

which is larger than 1 when $\lambda<1$. On the contrary, the smallest of the two roots is

$$
\frac{1-\sqrt{2 \lambda^{2}-1}}{2\left(1-\lambda^{2}\right)}
$$

and is smaller than 1 . It is larger than $1 / 2$ because $1-\sqrt{2 \lambda^{2}-1} \geq 1-\lambda^{2} \Leftrightarrow \lambda^{2} \geq \sqrt{2 \lambda^{2}-1}$ $\Leftrightarrow\left(1-\lambda^{2}\right)^{2} \geq 0$, which obviously holds. Finally notice that $P(1 / 2)=1 / 2\left(1-\lambda^{2}\right)>0$ and $P(1)=1-2 \lambda^{2}<0$ from our assumptions.

If $\lambda^{2}=1 / 2$ then there is a unique solution: $\alpha=1$. 


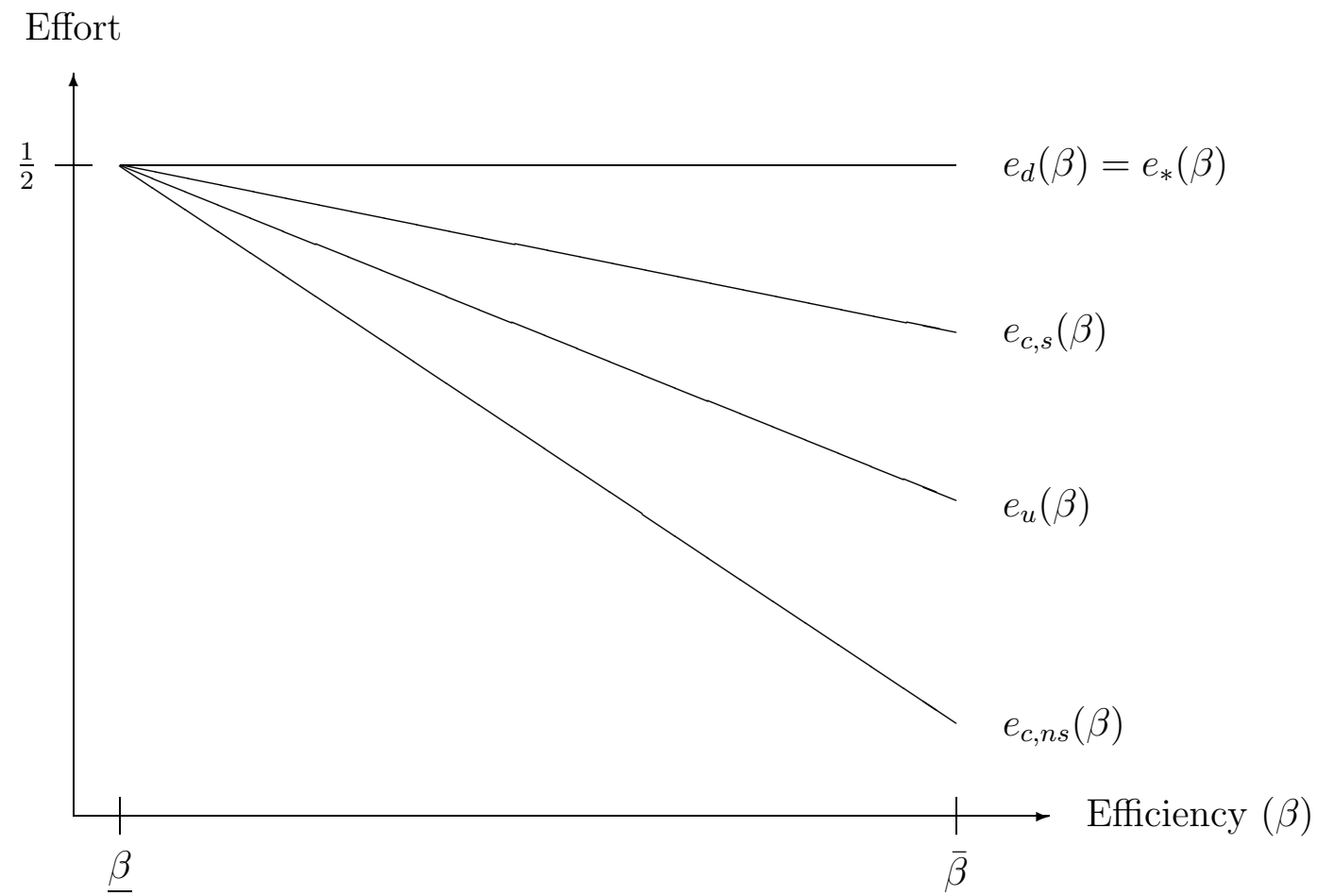

Figure 1: The profiles of effort. 